\title{
Übersichten
}

Ophthalmologe $2021 \cdot 118: 1013-1023$ https://doi.org/10.1007/s00347-020-01319-8 Eingegangen: 25 . September 2020 Überarbeitet: 17. Dezember 2020 Angenommen: 29. Dezember 2020 Online publiziert: 27. Januar 2021 (c) Der/die Autor(en) 2021
Isabel Bachmeier ${ }^{1}$ Christiane Blecha ${ }^{1}$ - Jürgen Föll ${ }^{2}$ - Daniel Wolff $\cdot$ Herbert Jägle

${ }^{1}$ Klinik und Poliklinik für Augenheilkunde, Universitätsklinikum Regensburg, Regensburg, Deutschland ${ }^{2}$ Abteilung für Pädiatrische Hämatologie, Onkologie und Stammzelltransplantation, Universitätsklinikum Regensburg, Regensburg, Deutschland

${ }^{3}$ Klinik und Poliklinik für Innere Medizin III, Universitätsklinikum Regensburg, Regensburg, Deutschland

\section{Makulopathie bei Sichelzellerkrankung}

Der nachfolgende Artikel wird Ihnen verraten, warum eine Erkrankung, die vorwiegend im „fernen Afrika“ anzutreffen ist und die roten Blutkörperchen betrifft, auch einem deutschen Augenarzt begegnen kann und deshalb auch Ihr Interesse wecken dürfte.

\section{Hintergrund}

\section{Epidemiologie, Pathogenese und klinische Manifestation der Sichelzellerkrankung}

Die Sichelzellerkrankung (SZE) zählt zu den weltweit häufigsten und klinisch bedeutsamsten erblichen Hämoglobinopathien, für welche ca. $5 \%$ der Weltbevölkerung Mutationsträger sind [48, 59]. Pro Jahr sind ca. 300.000 Neugeborene, davon $75 \%$ in subsaharischen Regionen Afrikas, betroffen. Weitere Endemiegebiete sind Indien, der Nahe Osten und der (vorwiegend östliche) Mittelmeerraum (u.a. Süditalien, Balkan, Griechenland, Türkei). Durch Migration aus endemischen Gebieten findet man auch in nördlicheren europäischen Ländern eine steigende Prävalenz der Erkrankung [59]. In Deutschland wird die aktuelle Prävalenz der SZE auf ca. 3000 geschätzt mit einer steigenden Tendenz durch Migranten aus Syrien, dem Irak und Zentralafrika [37, 38].

Bei der SZE handelt es sich um eine monogenetisch vererbte Hämoglobinopathie durch eine Punktmutation im $\beta$-Globin-Gen auf Chromosom 11p15.5. Der Austausch einer einzelnen Aminosäure $(\beta 6 \mathrm{Glu} \rightarrow \mathrm{Val})$ des normalen Hämoglobin HbA verändert die Eigen- schaften des so entstandenen anormalen Hämoglobin HbS derart, dass es bei Desoxygenierung polymerisiert [19]. Dies führt zur namensgebenden sichelförmigen Veränderung der Erythrozyten. Die sog. Sichelzellen sind fragil und weniger verformbar, was zu Hämolyse und rezidivierenden schmerzhaften vasookklusiven Krisen führt. Darüber hinaus werden durch Induktion von oxidativem Stress inflammatorische Prozesse in Gang gesetzt, woraus eine chronische Vaskulopathie resultiert. Diese kann die Mikrozirkulation aller Organsysteme betreffen und u. a. zu Niereninsuffizienz, Leberzirrhose, pulmonaler Hypertonie, ischämischen und hämorrhagischen ZNS-Infarkten oder Osteonekrosen führen [59]. Betroffene Patienten versterben häufig an kardiovaskulären, respiratorischen oder zerebrovaskulären Komplikationen. Der Genotyp beeinflusst den Phänotyp, wobei der homozygote HbSS-Genotyp und der gemischt heterozygote $\mathrm{HbS} \beta^{0}$ Thal $(\beta$-Thalassämie)Genotyp tendenziell mit schwereren klinischen Verläufen assoziiert sind als der gemischt heterozygote HbSC-Genotyp (HbC entsteht durch die Substitution $\beta 6$ Glu $\rightarrow$ Lys) [76].

Aufgrund des komplexen und weitgreifenden klinischen Bildes hat sich die Bezeichnung "Sichelzellerkrankung“ durchgesetzt. Nur der HbSS-Genotyp wird im englischen Sprachraum noch als „Sichelzellanämie“ bezeichnet.

\section{Okuläre Manifestation}

Die okuläre Manifestation umfasst u.a. kommaförmige Bindehautgefäße, ein
Sekundärglaukom durch Hyphäma oder Rubeosis iridis und orbitale Komplikationen $[18,57]$. Weitaus bekannter sind die retinalen Komplikationen, wobei man eine proliferative und eine nichtproliferative Sichelzellretinopathie (SZR) unterscheidet [77]. Unabhängig davon kann eine Makulopathie auftreten [46]. Zur Veranschaulichung dienen 2 Fallvorstellungen von Patienten mit SZE, bevor auf die verschiedenen Formen der retinalen Beteiligung bei SZE detailliert eingegangen wird.

\section{Fallvorstellung 1}

In der Augenklinik des Universitätsklinikum Regensburg wurde eine 20-jährige Patientin mit seit der Kindheit bekannter HbSS-SZE vor geplanter Stammzelltransplantation (HSCT [„hematopoietic stem cell transplantation"]) untersucht. Die Indikation zur HSCT wurde wegen zahlreicher Komplikationen der SZE mit teils schwerwiegenden Komplikationen an verschiedenen Organsystemen (multiple Schmerzkrisen, akute Thoraxsyndrome, Lungenembolien, ZNSIschämien, multiple Osteonekrosen) gestellt. Die Patientin war okulär beschwerdefrei mit einem unkorrigierten Visus von 1,0 beidseits. Spaltlampenmikroskopisch zeigten sich unauffällige Befunde mit klaren brechenden Medien. Die Fundoskopie ergab vitale Sehnerven mit unauffälliger zentraler und peripherer Netzhaut ( $\bullet$ Abb. 1a) ohne Hinweise auf eine Sichelzellretinopathie oder angioide Streifen. Überraschenderweise zeigte sich in der OCT eine Verdünnung der zentralen Netzhaut, die insbesonde- 

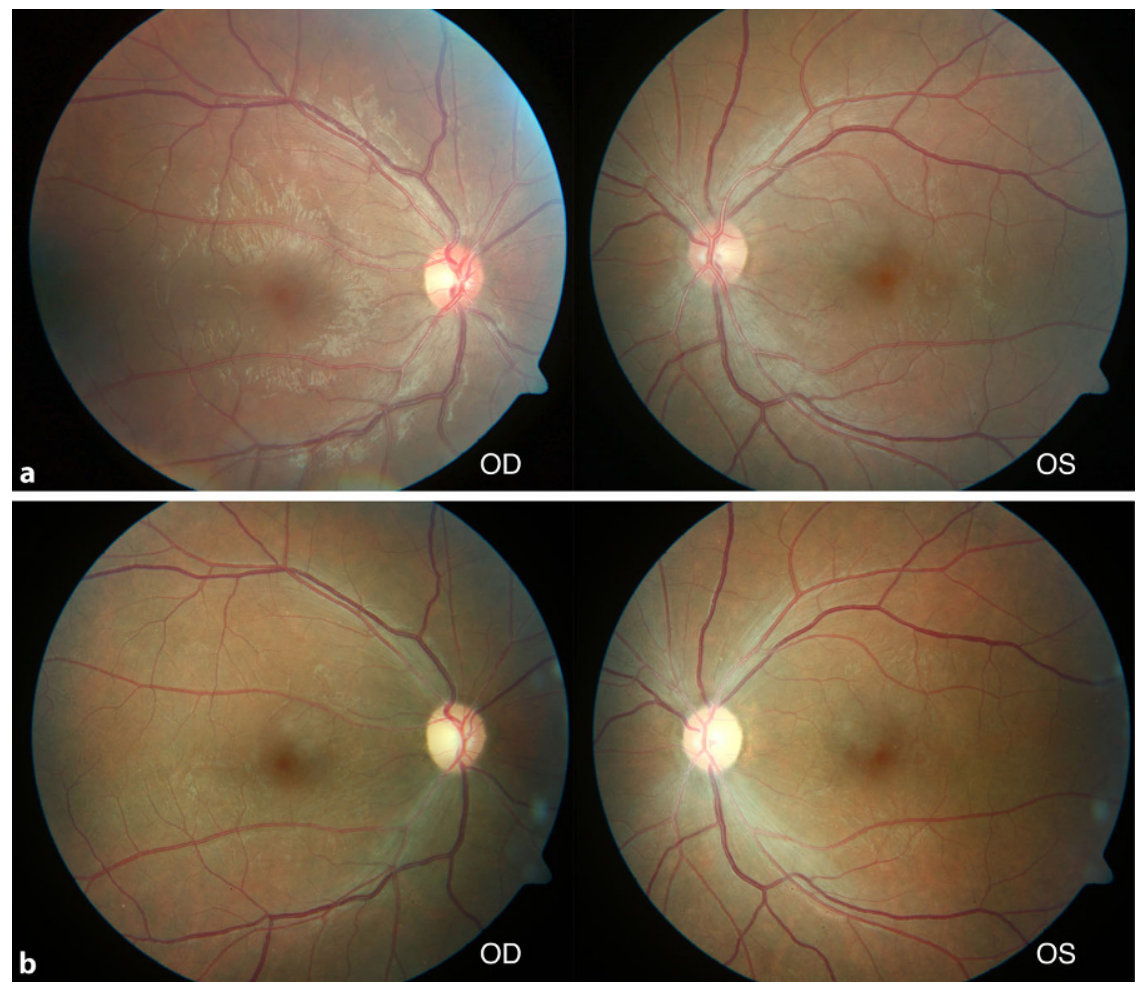

Abb. 1 \ Fall 1 - Farbfundusbilder beider Augen a einer 20-jährigen Patientin mit HbSS-Sichelzellerkrankung bei Erstvorstellung: es bestanden unauffällige fundoskopische Befunde und $\mathbf{b}$ nach $15 \mathrm{Mo}$ naten: die Makula entwickelte einen stumpfen Aspekt, und es trat eine beidseitige Papillenabblassung ein

re die inneren Netzhautschichten betraf und im temporalen Makulabereich besonders ausgeprägt war (• Abb. 2a). Aufgrund der allgemeinen Situation der Patientin musste auf Spezialuntersuchungen verzichtet werden. Die Patientin wies im weiteren Verlauf eine milde okuläre Graft-versus-Host-Disease (GvHD) auf, welche mit Tränenersatzmitteln und später auch Ciclosporin A AT (Ikervis ${ }^{\circledR}$, Santen GmbH, München, Deutschland) behandelt wurde, und berichtete von einer intermittierenden Visusminderung. Es bestanden leicht schwankende Visuswerte rechts zwischen 0,6 und 1,0 und links zwischen 0,8 und 1,0 , die 15 Monate später auf 0,5 abgefallen waren. Die Verdünnung der zentralen Netzhaut war fortgeschritten und betraf nun den gesamten Makulabereich (• Abb. 2b). Es zeigte sich fundoskopisch beidseits auch eine Papillenabblassung ( $\bullet \mathbf{A b b}$. 1b) mit Abnahme der retinalen Nervenfaserschichtdicke (RNFL) im Papillen-OCT (• Abb. 3). Im ergänzten Muster-ERG zeigten sich reduzierte Amplituden und im Muster-VEP keine reproduzierba- re Wellenform. Eine Literaturrecherche erbrachte schließlich eine Übereinstimmung der vorliegenden Veränderungen mit Beschreibungen der Sichelzellmakulopathie (SZM).

\section{Fallvorstellung 2}

Ein junger Patient (12 Jahre) hatte eimit multiplen Komplikationen (u.a. Schmerzkrisen, eine aplastische Krise nach Parvo-B19-Infektion und erste Hinweise einer Sichelzellnephropathie mit Proteinurie), weshalb er bereits mehrfach Bluttransfusionen erhalten hatte. Er war ebenfalls ophthalmologisch asymptomatisch und wies einen unkorrigierten Visus von beidseits 1,0 auf. Spaltlampenmikroskopisch zeigten sich kommaförmige Bindehautgefäße (• Abb. 4). Retinale Veränderungen (insbesondere Zeichen einer peripheren SZR oder angioide Streifen) bestanden nicht. Die OCT ergab insbesondere am rechten Auge eine temporale Makulaverdünnung, die in der OCT-A mit ne seit 10 Jahren bekannte HbSS-SZE nicht perfundierten Arealen im tiefen Kapillarplexus (DCP [„,deep capillary plexus"]) korrespondierte (• Abb.5).

\section{Sichelzellretinopathie}

Bei der SZR unterscheidet man proliferative und nichtproliferative Veränderungen [77]. Die von Goldberg 1971 etablierte Stadieneinteilung der proliferativen SZR (PSR) umfasst 5 Stadien (• Tab. 1; [25]). Die Stadien I und II stellen streng genommen nichtproliferative Stadien dar und bezeichnen periphere nicht perfundierte Netzhautareale (Stadium I) und die Ausbildung arteriovenöser Gefäßanastomosen (Stadium II). Das Stadium III ist durch Tiefseefächer-artige Neovaskularisationen (,sea fans") an der Grenze von vaskularisierter zu nicht vaskularisierter Netzhaut gekennzeichnet. Diese okkludieren und bilden sich im Gegensatz zu Proliferationen anderer Genese (z.B. diabetische Retinopathie) in einem hohen Prozentsatz spontan zurück (Autoinfarzierung)[16]. Sie können jedoch auch zur Glaskörperblutung (Stadium IV) oder traktionsbedingten bzw. rhegmatogenen Netzhautablösung (Stadium V) führen. Angaben zur Prävalenz der PSR schwanken stark zwischen verschiedenen Studien und sind von verschiedenen Faktoren abhängig. Generell nimmt die Prävalenz mit dem Alter zu [7, 42]. Patienten mit dem HbSC-Genotyp erleiden im Vergleich zum HbSS-Genotyp häufiger eine PSR und schwerere Verläufe einer PSR [42]. Die Schwere der PSR korreliert auch mit der Schwere des systemischen klinischen Verlaufs [26, 42].

Neben den genannten Befunden der PSR beinhaltet die retinale Beteiligung auch nichtproliferative Veränderungen wie sog. Lachsflecken („salmon patches"). Diese stellen meist unter der ILM gelegene durch Hämolyse lachsfarbene Blutungen dar, die spurlos abheilen können oder sog. Glitzerflecken (,iridescent spots") hinterlassen können. Es können aber auch durch reaktive fokale RPE-Hyperplasie und -Migration pigmentierte chorioretinale Narben, sog. schwarze Sonnenflecken („black sun bursts"), entstehen [21]. 
Es wurde auch ein gehäuftes Auftreten einer Tortuositas vasorum [70] und von "angioid streaks" bei SZE beschrieben [51].

\section{Sichelzellmakulopathie}

Bereits in den 70er-Jahren wurden makuläre Veränderungen im Rahmen einer SZE beschrieben. Romayanada et al. zeigten 1973 in histologischen Untersuchungen eine Verdünnung der inneren Netzhautschichten (Ganglionzell- und innere Körnerschicht) [63]. Im Jahr 1978 beschrieb Goldbaum mit dem Begriff „retinal depression sign" eine fundoskopisch anomale Reflektivität der ILM infolge einer perfusionsbedingten Verdünnung der inneren Netzhaut [24]. Auch fluoreszeinangiographisch wurden bei SZE Gefäßveränderungen im Makulabereich, eine vergrößerte foveoläre avaskuläre Zone und nicht perfundierte perifoveale Areale dargestellt $[3,5,70]$. Schon in diesen Arbeiten zeigte sich eine Prädilektion der temporalen Makula. Eine Erklärung lieferten Stevens et al., die die Gefäße im
Bereich der temporalen Raphe als Endgefäße beschrieben und dementsprechend die von ihnen perfundierten Netzhautareale als eine Art Wasserscheide der retinalen Perfusion [70].

Eine temporale Verdünnung der Makula in der SD-OCT wurde erstmals von Murthy et al. im Jahr 2011 bei asymptomatischen Patienten mit vollem Visus beschrieben [50]. Im selben Jahr veröffentlichten Hoang et al. eine gehäuft bei SZE-Patienten beobachtete zentrale Makulaverdünnung mit Aufweitung der fovealen Depression („foveal splaying“) [33]. Diese Veränderung wurde später von Mathew et al. jedoch eher als Normvariante bei Dunkelhäutigen und bei Patienten afrikanischer Abstammung gewertet [46].

Schließlich wurde, ebenfalls bei asymptomatischen Patienten, mittels OCT-A nachgewiesen, dass die Atrophie auf eine Perfusionsstörung vornehmlich im DCP zurückzuführen ist [30,31], also auf einer fokalen arteriolären Okklusion beruht. Es konnte gezeigt werden, dass auch asymptomatische Patienten in der (Mikro-)Pe- rimetrie zentrale und parazentrale Skotome [14] und Einschränkungen im Farbund Kontrastsehen [44] aufweisen. Hierbei korrespondierten die Gesichtsfeldbereiche herabgesetzter und fehlender Sensitivität mit Nonperfusionsarealen im DCP [65]. Auch elektrophysiologisch ließen sich bei asymptomatischen Patienten Makulafunktionsstörungen mittels multifokalem ERG nachweisen [8].

Aus einer Studie von Martin et al. geht hervor, dass die SZM bereits früh in der Kindheit auftritt [45] und damit deutlich früher als die periphere SZR, für die der Gipfel des Auftretens im Jugendoder frühen Erwachsenenalter liegt [7]. Die Prävalenz der SZM wird mit bis zu $64 \%$ der SZE-Patienten angegeben [45]. Sie betrifft Patienten mit HbSSSZE möglicherweise häufiger als Patienten mit HbSC-SZE [46], während es sich bei der PSR genau andersherum verhält [42]. Auch wenn eine temporale Makulaverdünnung unabhängig vom gleichzeitigen Vorliegen einer PSR auftreten kann, so scheint sie dennoch mit dem Grad peripherer Nonperfusion zu kor-

Hier steht eine Anzeige. 
relieren und mit einem höheren Risiko einer PSR vergesellschaftet zu sein [46]. Auch wurde das Vorliegen einer SZM mit einem vermehrten Auftreten von zerebrovaskulären Komplikationen assoziiert, wodurch die SZM trotz asymptomatischen Verlaufs und trotz fehlender Therapieoptionen klinische Relevanz erlangt [45].

\section{Optikusneuropathie und para- papilläre Nervenfaserschicht}

Zusätzlich zur Makulaverdünnung wies der von uns beschriebene Fall 1 im Verlauf eine Papillenabblassung mit Abnahme der parapapillären RNFL auf.

Vielfach wurde postuliert, dass Patienten mit SZE einem erhöhten Risiko unterliegen, ausgelöst durch einen $\mathrm{Au}$ geninnendruckanstieg eine Ischämie im Bereich des Sehnerven zu erleiden [27]. Zudem wurde ein erhöhtes Risiko für eine ischämische Optikopathie im Rahmen nichtokulärer Operationen beschrieben [64]. Es wurden auch Kasuistiken zu sich spontan entwickelnden AIONs [39] und PIONs [58, 69] veröffentlicht.

Darüber hinaus kann eine chronische Mikroangiopathie der den Sehnervkopf versorgenden Gefäße, vergleichbar mit der diabetischen Optikopathie, im Verlauf zur Papillenabblassung führen [68]. Bei SZE-Patienten ohne Glaukom wurde eine Abnahme der parapapillären RNFL mittels OCT beobachtet, deren Ausmaß mit der Schwere der Makulopathie [15] und deren Progredienz mit dem Vorhandensein zerebrovaskulärer Komplikationen [72] korrelierte. Auch für andere vaskuläre Retinopathien (Diabetes, arterieller Verschluss, HIV-Mikrovaskulopathie) wurde eine Abnahme der parapapillären RNFL beschrieben $[4,41,56]$.

\section{Differenzialdiagnostische Überlegungen}

\section{Medikamentennebenwirkungen}

Häufig bei SZE verabreichte Medikamente sind Substanzen aus der Gruppe der Chelatbildner. Bei wiederholt notwendigen Bluttransfusionen überschreitet die anfallende Menge an Eisen die Kapazität des Körpers, Eisen zu binden und

Ophthalmologe 2021 · 118:1013-1023 https://doi.org/10.1007/s00347-020-01319-8

(c) Der/die Autor(en) 2021

\section{Bachmeier · C. Blecha · J. Föll · D. Wolff · H. Jägle}

\section{Makulopathie bei Sichelzellerkrankung}

\section{Zusammenfassung}

Hintergrund. Die Sichelzellerkrankung (SZE)

ist eine hereditäre Hämoglobinopathie, die durch rezidivierende vasookklusive Episoden zur Mikrozirkulationsstörung verschiedener Organsysteme mit teils letalem Ausgang führt. Bei der okulären Manifestation der SZE ist am bekanntesten die periphere Sichelzellretinopathie (SZR). Unabhängig davon kann es bereits früh im Krankheitsverlauf zur Sichelzellmakulopathie (SZM) kommen.

Methoden. Review der internationalen und deutschsprachigen Literatur zur okulären Beteiligung bei SZE mit Fokus auf die SZR und SZM sowie Überblick über aktuelle systemische Therapieansätze bei SZE anlässlich der Vorstellung zweier Patienten mit HbSS-SZE.

Ergebnis und Schlussfolgerung. Im Gegensatz zur SZR ist die SZM mit temporaler
Verdünnung der inneren Netzhautschichten erst in den letzten 5 Jahren mit der Einführung von SD-OCT und OCTA vermehrt in die Literatur eingegangen. Unabhängig vom Vorliegen einer SZR kann es immerhin bei etwa der Hälfte der Patienten bereits früh im Krankheitsverlauf zu einer SZM kommen. Das Krankheitsbild wird auch in Deutschland durch den Fortschritt der systemischen Therapiemöglichkeiten und aufgrund von Migration präsenter werden. Durch Wissen um diese Komplikation der SZE kann eine frühzeitige Diagnosestellung erfolgen und unnötige Diagnostik vermieden werden.

\section{Schlüsselwörter}

Sichelzellmakulopathie · Makulaverdünnung $\cdot$ Retinopathie · Optikopathie · OCTAngiographie

\section{Maculopathy in sickle cell disease}

\section{Abstract}

Background. Sickle cell disease (SCD) is a hereditary hemoglobinopathy, which leads to microcirculatory disturbances of various organ systems through recurrent vaso-occlusive episodes, with a possibly fatal outcome. Sickle cell retinopathy $(\mathrm{SCR})$ is the best described ocular manifestation of SCD. Irrespective of the presence of peripheral SCR, sickle cell maculopathy (SCM) can occur early in the course of the disease.

Methods. Review of the international and German literature on ocular involvement in SCD with a focus on SCR and SCM and an overview of current systemic therapeutic approaches in SCD on the occasion of the presentation of two patients with $\mathrm{HbSS}$ SCD. Results and conclusion. In contrast to SCR, $\mathrm{SCM}$ with temporal thinning of the inner retinal layers has only been increasingly described in the literature in the last 5 years, with the advent of SD-OCT and OCTA. Irrespective of the presence of SCR, as many as about half of the patients may develop SCM early in the course of the disease. As a result of progress in systemic therapeutic options and due to migration, the clinical picture will occur more often also in Germany. By knowing about this complication of SCD an early diagnosis can be made and unnecessary diagnostics can be avoided.

\section{Keywords}

Sickle cell maculopathy - Macular thinning · Retinopathy · Optic neuropathy · OCT angiography auszuscheiden. Der potenziell für sämtliche Körperzellen toxische erhöhte Spiegel ungebundenen Eisens kann durch Verabreichung chelatbildender Substanzen gebunden und über den Urin oder die Fäzes ausgeschieden werden. Verfügbare Eisenchelatoren sind Deferoxamin und Deferasirox [54].

In Zusammenhang mit dem seit fast 60 Jahren eingesetzten Deferoxamin wur- den RPE-Veränderungen teils mit Akkumulation von Material im Bereich der äußeren Retina, eine Schießscheibenmakulopathie, eine Verdickung der BruchMembran und seltener eine pseudovitelliforme Makulopathie beschrieben [10].

Für das seit 2002 verfügbare Deferasirox wurden milde makuläre Pigmentveränderungen [75], eine perifoveale Verdünnung der äußeren Netzhaut mit Un- 

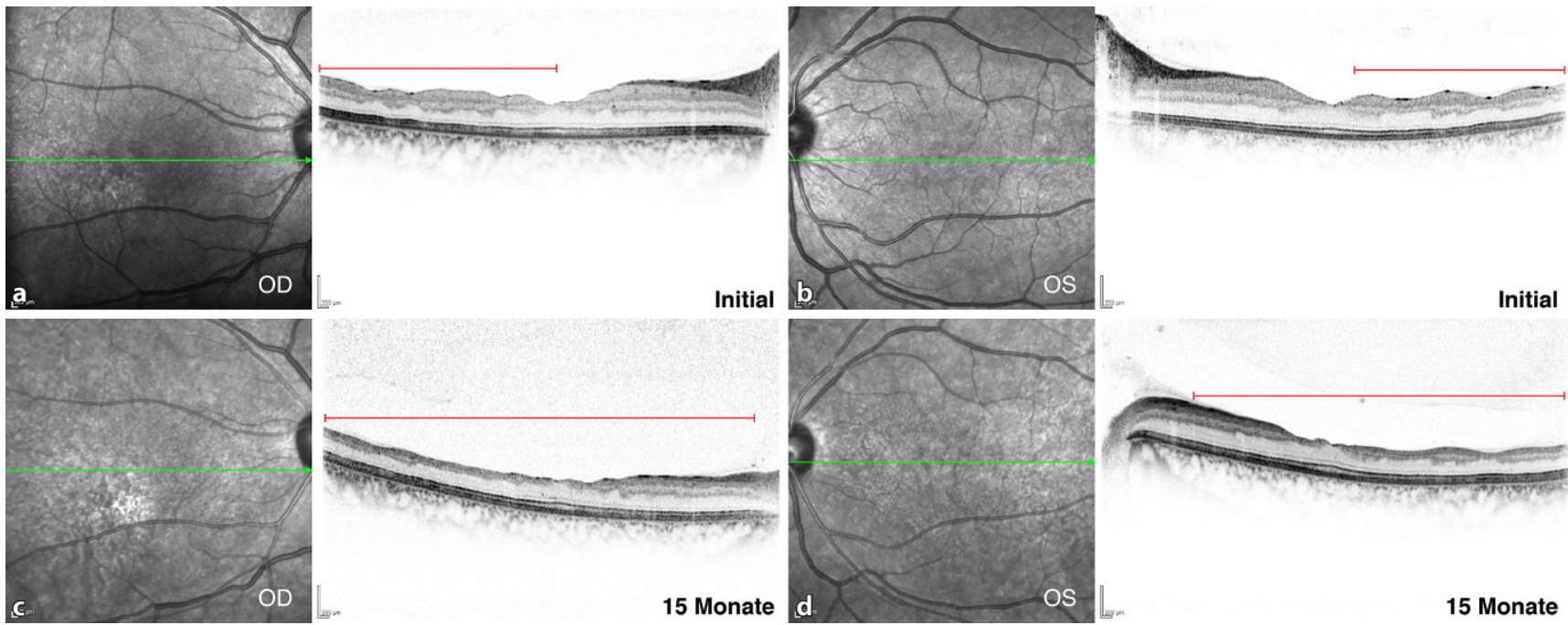

Initial

Abb. 2 \ Fall 1 - Optische Kohärenztomographie des rechten $(\mathbf{a}, \mathbf{c})$ und linken Auges $(\mathbf{b}, \mathbf{d})$. Eine initial temporal betonte Verdünnung der inneren Netzhautschichten $(\mathbf{a}, \mathbf{b})$ dehnte sich im Verlauf auf den gesamten Makulabereich aus ( $\mathbf{c}, \mathbf{d})$. Die roten Balken markieren die Bereiche verdünnter Netzhaut

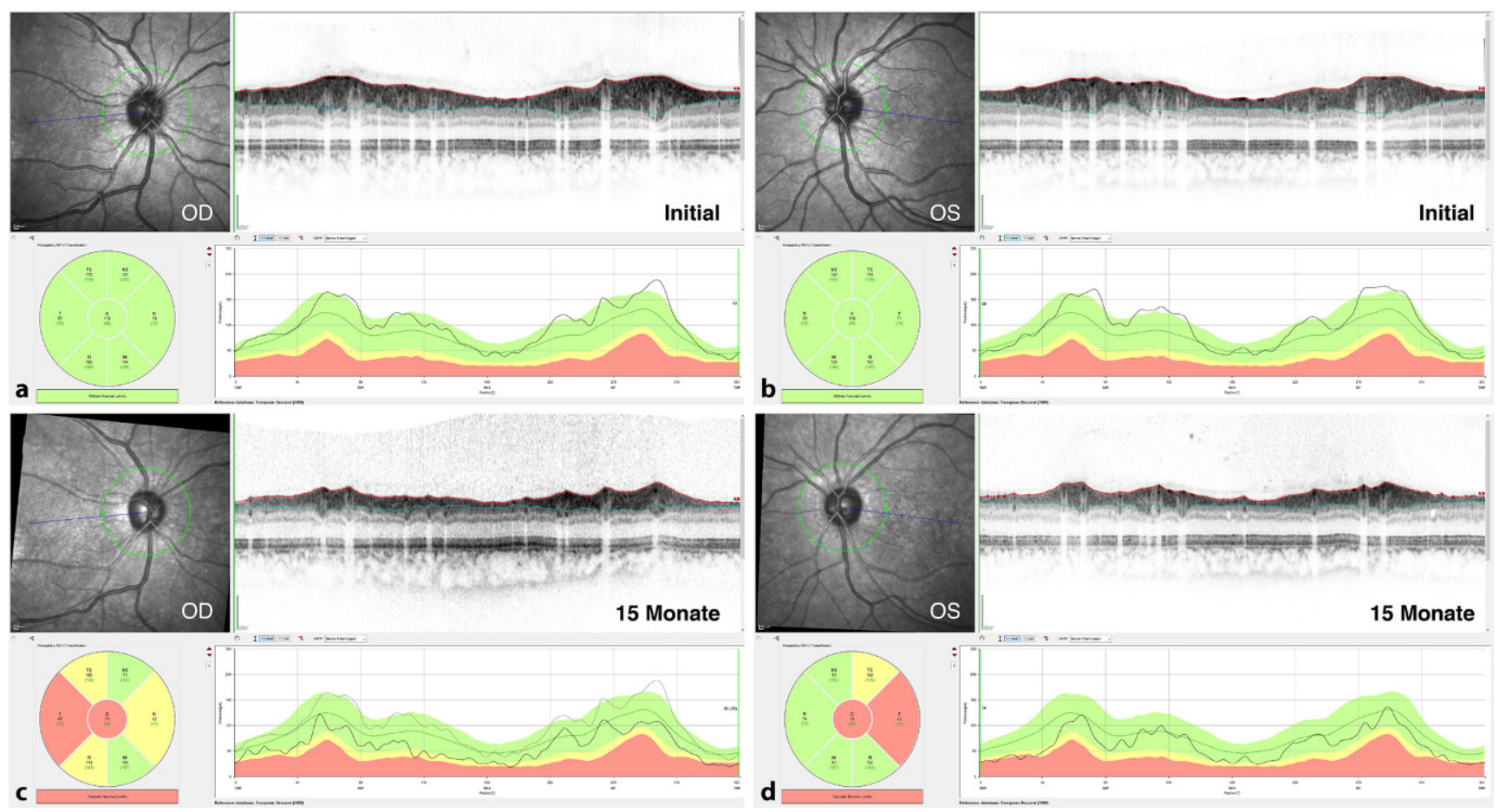

Abb. $3 \Delta$ Fall 1 - Optische Kohärenztomographie der Papillen des rechten $(\mathbf{a}, \mathbf{c})$ und linken Auges $(\mathbf{b}, \mathbf{d})$. Die retinale Nervenfaserschichtdicke lag initial in allen Sektoren im Normbereich $(\mathbf{a}, \mathbf{b})$ und nahm im Verlauf betont in den temporalen Sektoren $\mathrm{ab}(\mathbf{c}, \mathbf{d})$

terbrechung der ellipsoiden Zone ähnlich frühen OCT-Veränderungen unter Hydroxychloroquin-Einnahme [54] und eine Zunahme einer bereits vorbestehenden Deferoxamin-induzierten pseudovitelliformen Makulopathie nach Wechsel auf Deferasirox [10] beschrieben. Die Häufigkeit des Auftretens einer Makulo- pathie wird in der Fachinformation mit 1:1000 bis 1:100 angegeben.

Die Deferoxamin- und Deferasiroxassoziierten Veränderungen und Funktionsstörungen sind nach Absetzen und Dosisreduktion teilweise reversibel. Diskutiert wird, dass die Schädigung auf einem direkten toxischen Effekt auf das RPE beruht oder indirekt auf eine Che- latbildung mit für das RPE essenziellen Spurenelementen (Zink, Kupfer) zurückzuführen ist [10]. Während bei der Makulopathie durch Chelatbildner Veränderungen im Bereich der äußeren Netzhaut teils mit Materialablagerung im Vordergrund stehen, ist die SZM durch eine Verdünnung der inneren Netzhautschichten ohne Ablagerungen gekennzeichnet. 


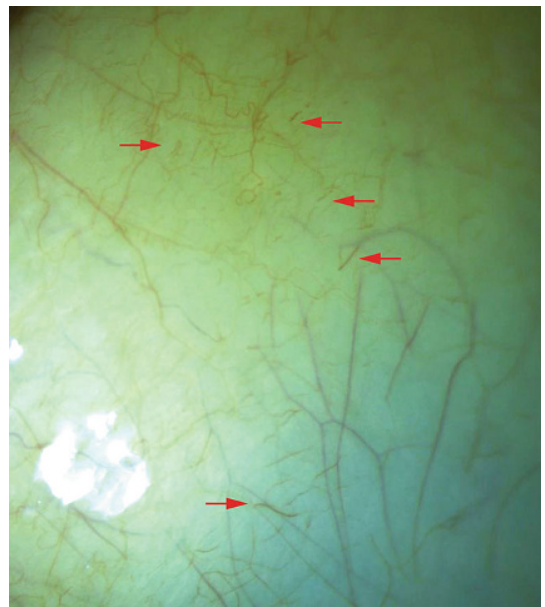

Abb. 4 \ Fall 2 - Vorderabschnittsfoto des linken Auges eines 12-jährigen Patienten mit HbSS-Sichelzellerkrankung. Die Pfeile zeigen auf die charakteristischen kommaförmigen Bindehautgefäße

Abraham et al. postulierten zudem, dass auch eine Eisen- und Ferritinablagerung selbst durch einen toxischen Effekt zur irreversiblen Schießscheibenmakulopathie führen kann. Die Annahme stützt sich jedoch auf eine Einzelfallbeobachtung [2].

Auch andere bei SZE eingesetzte Medikamente können Makulopathien verursachen. Ding et al. fanden histologisch eine Atrophie der inneren Netzhaut mit einem massiven Untergang retinaler Ganglienzellen nach Einnahme des Purinanalogons Fludarabin, welches als Zytostatikum zur Vorbereitung vor HSCT eingesetzt wird [17]. Die Patienten hatten jedoch eine akute Visusminderung über wenige Wochen bis auf Lichtscheinwahrnehmung bzw. fehlende Lichtscheinwahrnehmung erlitten.

Für das Antimykotikum Fluconazol wurde die Entstehung eines zystoiden Makulaödems nach 1-jähriger oraler Einnahme beschrieben [43]. Ein Makulaödem konnte bei der von uns in Fall 1 vorgestellten Patientin auch nach Einnahme zu keinem Zeitpunkt festgestellt werden.

\section{Retinale Komplikationen nach HSCT}

Nach HSCT entwickeln bis zu $10 \%$ der Patienten eine retinale Mikroangiopathie, die auch eine Netzhautischämie
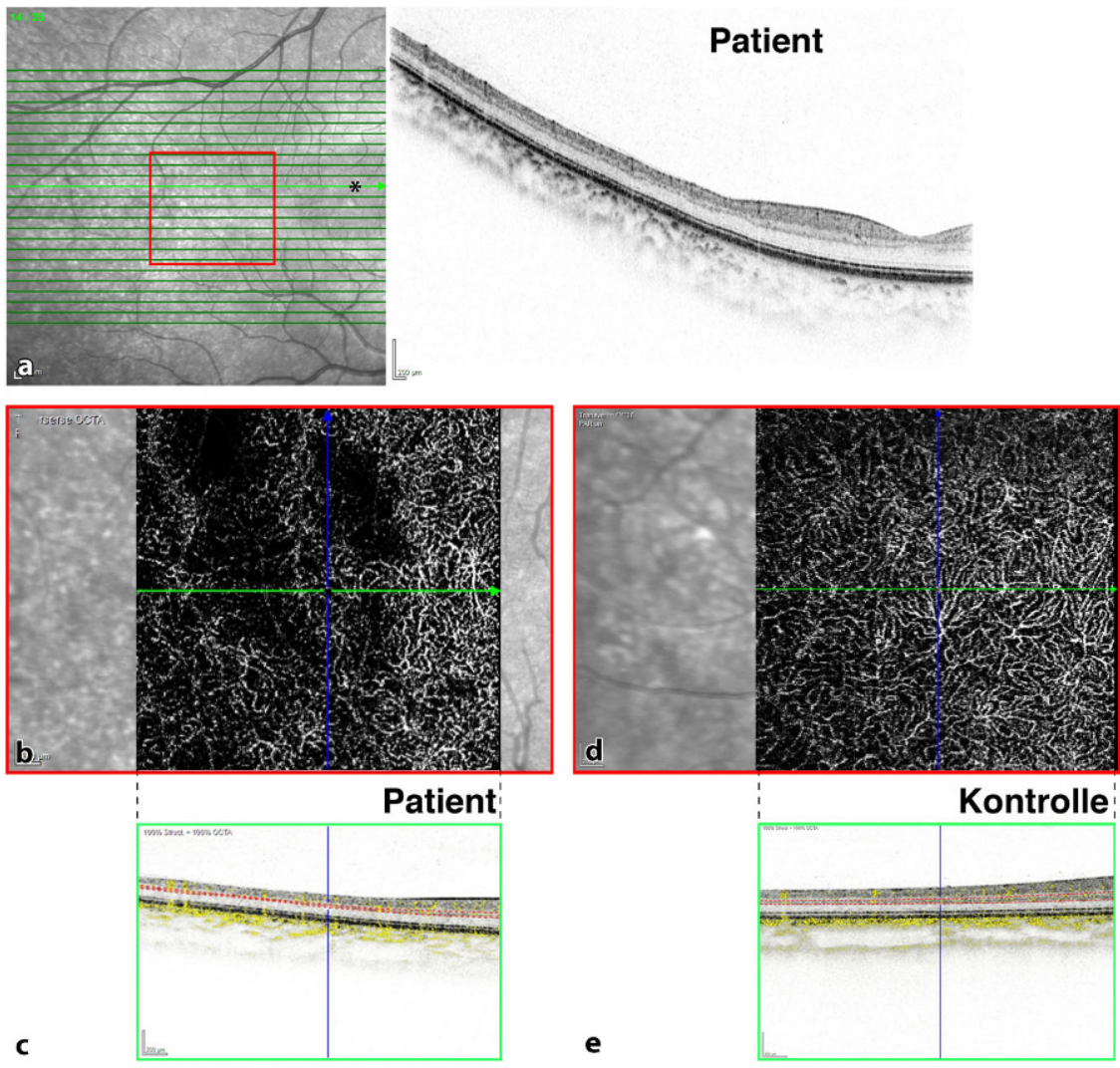

Abb. 5 \ Fall 2 - Optische Kohärenztomographie-Schnittbild (a) des rechten Auges des Jungen aus Fall 2 mit deutlicher Verdünnung der Netzhaut (Stern markiert die Foveola). Der rot gerahmte Bereich entspricht dem in (b) mit der optischen Kohärenztomographie-Angiographie (OCT-A) untersuchten Netzhautareal. Der Bereich verdünnter Netzhaut korrespondiert mit Nonperfusionsarealen im tiefen Kapillarplexus $(D C P)$. Die Lage des DCP ist im zugehörigen Schnittbild (c) mit gestrichelten roten Linien illustriert. Im Vergleich dazu die OCT-A des entsprechenden Bereichs einer 17-jährigen gesunden Kontrollperson ohne Perfusionsstörung im DCP (d) sowie die Lage des DCP im zugehörigen Schnittbild (e)

beinhalten kann [36]. Ursächlich ist eine Kombination aus Grunderkrankung, vorbereitender Therapie (Zytostatika, Bestrahlung) und GvHD. In der Regel zusätzlich vorliegende intraretinale Blutungen, Mikroaneurysmata, Cottonwool-Herde oder harte Exsudate wurden bei der vorgestellten Patientin (Fall 1) zu keinem Zeitpunkt beobachtet. Die HSCT kann jedoch das rasche Fortschreiten der Makulaatrophie genauso wie die sich entwickelnde Papillenabblassung begünstigt haben.

Es werden im Zusammenhang mit HSCT selten Optikusneuropathien beschrieben wie auch bei der retinalen Mikroangiopathie auf dem Boden der Grunderkrankung, der vorbereitenden potenziell neurotoxischen Medikamente (wie das bereits erwähnte Zytostatikum Fludarabin) und der GvHD [36].

\section{Zustand nach manifestem Arterienastverschluss}

Der Verschluss eines makulaversorgenden Arterienasts führt nach einem initialen Nervenfaserödem im Verlauf ebenfalls zur Atrophie und Verdünnung im Bereich der inneren Netzhautschichten [41]. In der Regel ist diese dann aber nicht asymptomatisch, sondern mit einem anamnestisch akuten Visusverlust und Zentralskotom vergesellschaftet [32].

\section{Parazentrale akute mittlere Makulopathie (PAMM)}

Der erstmals 2013 beschriebenen PAMM liegt ein Verschluss im Bereich des DCP zugrunde, sie ist assoziiert mit retinalen Gefäßerkrankungen, und als Auslöser gelten u. a. systemische Hypovolämie, virale Infekte, Migräne und Trau- 
ma [61]. Während in der OCT initial eine fokale bandförmige Hyperreflektivität vorwiegend auf Höhe der inneren Körnerschicht besteht, entwickelt sich innerhalb von Monaten an dieser Stelle eine Verdünnung der inneren Körnerschicht und der äußeren plexiformen Schicht, begleitet von einer Verdickung der äußeren Körnerschicht. Insgesamt resultiert eine fokale Exkavation der inneren Netzhautoberfläche [52]. Im Allgemeinen ist sie im Akutstadium mit einer umschriebenen flauen gräulichen Fundusaufhellung verbunden und wird mit dem akuten Auftreten eines Skotoms symptomatisch. Aufgrund des identischen Pathomechanismus (Verschluss im DCP) und der späteren Entwicklung einer Atrophie im Bereich der inneren Netzhaut wird die PAMM von manchen Autoren sogar als die der SZM zugrunde liegende Vorläuferläsion diskutiert [34].

\section{Alport-Syndrom}

Das Alport-Syndrom ist eine hereditäre Basalmembranerkrankung, der ein Strukturdefekt von Kollagen-IV-Fasern zugrunde liegt und die vorwiegend die renalen Glomeruli betrifft. Sie geht mit einem progressiven Nierenversagen einher, häufig besteht eine Assoziation mit einer progredienten Innenohrschwerhörigkeit [29]. Die okuläre Manifestation umfasst neben einem Lenticonus anterior, einer Katarakt, kornealen Trübungen und retinalen Flecken auch eine Makulopathie [67]. Es wurde eine Verdünnung der temporalen Makula beschrieben, wie bei der SZM überwiegend im Bereich der inneren Netzhautschichten, wobei diese aber gleichmäßiger verdünnt sind als bei der SZM [66, 74]. Eine unregelmäßige Verdünnung der inneren Netzhautschichten bei ebenfalls beschriebenen Makulaschichtforamina ähnelt im Aspekt eher der SZM, ist aber auf die Fovea beschränkt [67].
Hier steht eine Anzeige. Springer 
Tab. 1 Stadieneinteilung der proliferativen Sichelzellretinopathie (PSR) nach Goldberg [25]

\section{Stadium I}

Periphere arterioläre Okklusionen

Stadium II

Arteriovenöse Anastomosen

Stadium III

Neovaskularisationen (,sea fans“)

Stadium IV

Glaskörperblutung

Stadium V

\section{Ophthalmologische Therapie- optionen}

\section{Behandlung bei erhöhtem Augeninnendruck}

Die Ausbildung eines Hyphämas oder einer Rubeosis iridis kann zu einem Sekundärglaukom und aufgrund der ohnehin erhöhten Anfälligkeit für Gefäßverschlüsse rasch zur ischämischen Optikusneuropathie oder zum Zentralarterienverschluss führen [27]. Ob bereits ein Augeninnendruck von $25 \mathrm{mmHg}$ Anlass für chirurgische Interventionen (z. B. Parazentese und Vorderkammerspülung bei Hyphäma) geben sollte, ist jedoch umstritten [40]. Wichtig ist es, Carboanhydrasehemmer zu vermeiden, da die damit einhergehende systemische Dehydratation bzw. Azidose zu erhöhter Blutviskosität in der Mikrozirkulation führen bzw. die Sichelzellbildung verstärken kann [20]. Dass dies auch für topisch applizierte Carboanhydrasehemmer (Dorzolamid, Brinzolamid) gelten könnte, ist denkbar - die Augentropfen könnten lokal in der Vorderkammer bei Hyphäma eine Sichelzellbildung verursachen mit Behinderung des Durchtritts der Sichelzellen durch das Trabekelmaschenwerk-, konnte bisher jedoch nicht nachgewiesen werden [40].

\section{Laserbehandlung}

Ziel einer Laserung ist es, eine Glaskörperblutung oder Traktionsablatio, also eine Progression der PSR zu Stadium IV bzw. V, zu verhindern. Frühere Ansätze, die „feeder vessels" der Neovaskularisationsmembranen direkt zu koagulieren, wurden mittlerweile verlassen, da dies potenziell eine Glaskörperblutung, Netzhautrisse oder choroidale Neovaskularisationen verursachen kann. Vielmehr empfiehlt man bei „sea fans“ mit ausbleibender spontaner Autoinfarzierung eine zirkuläre disseminierte Photokoagulation oder eine gezielte Laserung im Bereich der ischämischen Netzhautareale [62].

\section{Anti-VEGF-Therapie}

Auch eine intravitreale Anti-VEGF-Therapie kann möglicherweise die Rückbildung von Neovaskularisationen und Glaskörperblutungen bewirken, ggf. auch als präoperative Eingabe vor einer geplanten $\mathrm{ppV}[11,47,49]$. Allerdings gibt es keine klinischen Studien, die eine Anti-VEGF-Therapie mit einer Scatterlaser-Therapie vergleichen. Als mögliche Komplikation einer Anti-VEGFTherapie wurde die Entwicklung eines Hyphäma beschrieben [6].

\section{Pars-plana-Vitrektomie}

Eine $\mathrm{ppV}$ ist bei nicht aufklarender Glaskörperblutung oder Ablatio indiziert. Es wurde hierbei eine erhöhte Rate von Ablatiorezidiven, iatrogenen Foramina und postoperativen Hyphämata oder Vorderabschnittischämien beschrieben [13]. Zur Dissektion der Neovaskularisationsmembranen wird eher zu Segmentations- als zu Delaminationstechniken geraten, da bei Letzteren aufgrund stark adhärenter Membranen ein erhöhtes Risiko für iatrogene Netzhautrisse besteht [78].

Die PSR wird im Allgemeinen nur selten therapiebedürftig, zugleich liegen keine klaren therapeutischen Leitlinien oder Empfehlungen aufgrund schwacher Evidenz vor. Einen umso höheren Stellenwert hat die Behandlung der Grunderkrankung. Diese kann auch einem Auftreten retinaler Komplikationen bzw. schwerer Verläufe zuvorkommen [1].

\section{Systemische Therapieoptionen}

Das einzige krankheitsmodifizierende Medikament in Europa ist Hydroxycarbamid, das ab dem 2. Lebensjahr zugelassen ist (nach neuen Leitlinien empfohlen bereits ab 9. Lebensmonat). Unter anderem induziert es eine vermehrte Bildung von fetalem Hämoglobin $(\mathrm{HbF})$, welches anders als das mutierte HbS nicht polymerisiert, sodass die Deformierung der Erythrozyten verhindert wird. Hierdurch wird die mediane Inzidenz schmerzhafter Krisen pro Jahr um $44 \%$ reduziert $[12,60]$. (Austausch-)Transfusionen reduzieren die Konzentration an HbS und sind zur Behandlung bestimmter Akutkomplikationen erforderlich, können aber auch Teil eines langfristig ausgelegten Dauertherapiekonzepts v.a. bei Patienten mit ZNS-Komplikationen in Kombination mit Hydroxycarbamid sein [9]. In Erprobung sind mehrere neuere Medikamente, die die pathophysiologischen Mechanismen der Vasookklusion bei SZE lindern sollen, wie z.B. Voxelotor, Crizanlizumab und Rivipansel, die die Häufigkeit von Vasookklusion, Krankenhausaufenthalt und Schmerzmittelkonsum zu verringern scheinen [53, 73].

Zurzeit ist die allogene hämatopoetische Stammzelltransplantation (HSCT) für Patienten mit schwerwiegenden Komplikationen der SZE (Schlaganfälle, akute Thoraxsyndrome, signifikante andere Organschäden) die einzige angebotene kurative Therapieoption. Die myeloablative HSCT mit Knochenmark eines HLA-identischen Geschwisterspenders (MSD) ist derzeit Standard der Behandlung mit einem Gesamtüberleben von $90 \%$, ereignisfreiem Überleben von $80 \%$ bei einer behandlungsbedingten Mortalität von $7 \%[23,55]$. Jedoch liegt die allgemeine Verfügbarkeit von MSD bei unter $14 \%$, und die Wahrscheinlichkeit, einen passenden nichtverwandten Fremdspender (MUD) zu finden, liegt bei SZE-Patienten afrikanischer Herkunft unter $18 \%$ [28, 71]. Teilweise HLAnicht-übereinstimmende Spender ersten Grades (sog. haplo-HSCT) können den Spenderpool auf durchschnittlich 2,7 pro Patient erhöhen [22]. 
Eine weitere Therapieoption ist die Gentherapie, bei der eigene (autologe) hämatopoetische Stammzellen des Patienten gewonnen und im Labor genetisch modifizierte $\beta$-Ketten $\left(\mathrm{HbA}^{\mathrm{T} 87 \mathrm{Q}}\right.$, „gene addition") mithilfe eines lentiviralen Vektors dauerhaft im Genom integriert werden [35]. Die korrigierten Zellen werden dem Patienten dann nach einer myeloablativen Chemotherapie wieder zugeführt. Theoretisch ist ein kurativer Ansatz durch eine homologe Rekombination ("gene correction“), also durch einen Austausch der Nukleotide, möglich. Dieser Goldstandard befindet sich noch in der vorklinischen Entwicklung. Eine weitere sehr interessante Methode ist die Induktion von $\mathrm{HbF}$ durch eine sog. "Genschere“. Dabei wird genomische DNA mit hoher Präzision modifiziert, um Insertionen sowie Deletionen (Indels) in die Erythroid-Enhancer-Region von BCL11A zu induzieren. Der Transkriptionsfaktor BCL11A, ein Regulator der HbF-Expression, unterdrückt die Expression der $\gamma$-Globin-Kette (HBG1, HBG2) innerhalb des HBB-Genclusters. Patienten mit natürlicher Variante von BCL11A exprimieren ungewöhnlich viel HbF-Ketten. Bei transfusionsabhängiger $\beta$-Thalassämie kann eine Hochregulierung von $\mathrm{HbF}$ die Notwendigkeit einer Transfusionstherapie verringern oder beseitigen, und bei der SZE könnten vasookklusive Krisen dadurch verringert werden [35]. Trotz vielversprechender Ergebnisse aus laufenden klinischen Studien fehlen für die Gentherapie noch Langzeitbeobachtungen über die Wirksamkeit und das Auftreten möglicher Nebenwirkungen.

Aufgrund von Migration aus endemischen Gebieten und aufgrund der angebotenen breiten Palette auch neuartiger Therapieoptionen werden zunehmend Patienten mit SZE in Deutschland behandelt werden. Daher wird auch deutschen Ophthalmologen das Krankheitsbild der SZR und SZM vermehrt begegnen, sodass es sich auch hierzulande lohnt, sich mit dieser interessanten Erkrankung auseinanderzusetzen.

\section{Fazit für die Praxis}

- Die Sichelzellerkrankung (SZE) zählt zu den häufigsten Hämoglobinopathien.

- Durch zunehmende Migration aus Endemiegebieten wird die SZE in Deutschland präsenter werden und auch Ophthalmologen vermehrt begegnen.

- Retinale Komplikationen der SZE umfassen periphere nichtproliferative und proliferative Veränderungen sowie eine Makulopathie (SZM).

- Die SZM ist durch eine temporale Verdünnung der inneren Netzhautschichten gekennzeichnet. Sie findet durch Fortschritt der apparativen Diagnostik erst in den letzten 5 Jahren vermehrt Eingang in die internationale Literatur.

- Wissen um diese Form der okulären Komplikation der SZE verhindert durch eine frühzeitige Diagnosestellung unnötige Diagnostik.

\section{Korrespondenzadresse}

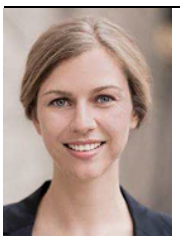

Dr. med. Isabel Bachmeier Klinik und Poliklinik für Augenheilkunde, Universitätsklinikum Regensburg Franz-Josef-Strauß-Allee 11, 93053 Regensburg, Deutschland isabel.bachmeier@mac.com

Funding. Open Access funding enabled and organized by Projekt DEAL.

\section{Einhaltung ethischer Richtlinien}

Interessenkonflikt. I. Bachmeier, C. Blecha, J. Föll, D. Wolff und $H$. Jägle geben an, dass kein Interessenkonflikt besteht.

Für diesen Beitrag wurden von den Autoren keine Studien an Menschen oder Tieren durchgeführt. Für die aufgeführten Studien gelten die jeweils dort angegebenen ethischen Richtlinien.

Open Access. Dieser Artikel wird unter der Creative Commons Namensnennung 4.0 International Lizenz veröffentlicht, welche die Nutzung, Vervielfältigung, Bearbeitung, Verbreitung und Wiedergabe in jeglichem Medium und Format erlaubt, sofern Sie den/die ursprünglichen Autor(en) und die Quelle ordnungsgemäß nennen, einen Link zur Creative Commons Lizenz beifügen und angeben, ob Änderungen vorgenommen wurden.

Die in diesem Artikel enthaltenen Bilder und sonstiges Drittmaterial unterliegen ebenfalls der genannten Creative Commons Lizenz, sofern sich aus der Abbildungslegende nichts anderes ergibt. Sofern das betreffende Material nicht unter der genannten Creative Commons Lizenz steht und die betreffende Handlung nicht nach gesetzlichen Vorschriften erlaubt ist, ist für die oben aufgeführten Weiterverwendungen des Materials die Einwilligung des jeweiligen Rechteinhabers einzuholen.

Weitere Details zur Lizenz entnehmen Sie bitte der Lizenzinformation auf http://creativecommons.org/ licenses/by/4.0/deed.de.

\section{Literatur}

1. Abdalla Elsayed MEA, Mura $\mathrm{M}, \mathrm{Al}$ Dhibi $\mathrm{H}$ et al (2019) Sickle cell retinopathy. A focused review. Graefes Arch Clin Exp Ophthalmol 257:1353-1364. https://doi.org/10.1007/s00417-019-04294-2

2. Abraham A (2018) Bull's eye maculopathy possibly due to iron overload in a child with thalassemia major: a case of possible "ferritin retinopathy". Retin Cases Brief Rep. https://doi.org/10.1097/ICB 0000000000000834

3. Acacio I, Goldberg MF (1973) Peripapillary and macular vessel occlusions in sickle cell anemia. Am J Ophthalmol 75:861-866. https://doi.org/10. 1016/0002-9394(73)90892-1

4. Arantes TE, Garcia CR, Tavares IM et al (2012) Relationship between retinal nerve fiber layer and visual field function in human immunodeficiency virus-infected patients without retinitis. Retina 32:152-159. https://doi.org/10.1097/IAE. Ob013e31821502e1

5. Asdourian GK, Nagpal KC, Busse B et al (1976) Macular and perimacular vascular remodelling sickling haemoglobinopathies. Br J Ophthalmol 60:431-453. https://doi.org/10.1136/bjo.60.6.431

6. Babalola OE (2010) Intravitreal bevacizumab (avastin) associated with secondary hyphaema in a case of proliferative sickle cell retinopathy. BMJ Case Rep 2010:bcr1120092441. https://doi.org/ 10.1136/bcr.11.2009.2441

7. Ballas SK, Kesen MR, Goldberg MF et al (2012) Beyond the definitions of the phenotypic complications of sickle cell disease: an update on management. Sci World J. https://doi.org/10. 1100/2012/949535

8. Beral L, Romana M, Lemonne $\mathrm{N}$ et al (2019) Multifocal electroretinogram findings in sickle cell maculopathy. Eye (Lond) 33:1939-1945. https:// doi.org/10.1038/s41433-019-0499-7

9. Brousse V, Gandhi S, de Montalembert M et al (2013) Combined blood transfusion and hydroxycarbamide in children with sickle cell anaemia. Br J Haematol 160:259-261. https://doi. org/10.1111/bjh.12104

10. Bui K (2017) Pseudovitelliform maculopathy associated with deferoxamine toxicity: multimodal imaging and electrophysiology of a rare entity. Digit JOphthalmol 23:11. https://doi.org/10.5693/ djo.02.2016.12.001

11. Cai CX, Linz MO, Scott AW (2018) Intravitreal bevacizumab for proliferative sickle retinopathy: a case series. J Vitreoretin Dis 2:32-38. https://doi. org/10.1177/2474126417738627

12. CharacheS, Terrin ML, Moore RD etal (1995) Design of the multicenter study of hydroxyurea in sickle 
cell anemia. Control Clin Trials 16:432-446. https:// doi.org/10.1016/S0197-2456(95)00098-4

13. Chen RWS, Flynn HW, Lee W-H et al (2014) Vitreoretinal management and surgical outcomes in proliferative sickle retinopathy: a case series. Am J Ophthalmol 157:870-875.e1. https://doi.org/10. 1016/j.ajo.2013.12.019

14. Chow CC, Genead MA, Anastasakis A et al (2011) Structural and functional correlation in sickle cell retinopathy using spectral-domain optical coherence tomography and scanning laser ophthalmoscope microperimetry. Am J Ophthalmol 152:704-711.e2. https://doi.org/10. 1016/j.ajo.2011.03.035

15. Chow CC, Shah RJ, Lim Jl et al (2013) Peripapillary retinal nerve fiber layer thickness in sicklecell hemoglobinopathies using spectral-domain optical coherence tomography. Am J Ophthalmol 155:456-464.e2. https://doi.org/10.1016/j.ajo. 2012.09.015

16. Condon PI, Serjeant GR (1980) Behaviour of untreated proliferative sickle retinopathy. $\mathrm{Br} J$ Ophthalmol 64:404-411. https://doi.org/10.1136/ bjo.64.6.404

17. Ding $X$, Herzlich AA, Bishop R et al (2008) Ocular toxicity of fludarabine: a purine analog. Expert Rev Ophthalmol 3:97-109. https://doi.org/10.1586/ 17469899.3.1.97

18. Do BK, Rodger DC (2017) Sickle cell disease and the eye. Curr Opin Ophthalmol 28:623-628. https:// doi.org/10.1097/ICU.0000000000000423

19. Eaton WA, Hofrichter J (1990) Sickle cell hemoglobin polymerization. Adv Protein Chem 40:63-279

20. Finch CA (1972) Pathophysiologic aspects of sickle cell anemia. Am J Med 53:1-6. https://doi.org/10. 1016/0002-9343(72)90110-6

21. Gagliano DA, Goldberg MF (1989) The evolution of salmon-patch hemorrhages in sickle cell retinopathy. Arch Ophthalmol 107:1814-1815. https://doi. org/10.1001/archopht.1989.01070020896034

22. Gladstone DE, Zachary A, Fuchs EJ et al (2011) Desensitization for mismatched hematopoietic stem cell transplantation (HSCT). Blood 118:1955-1955. https://doi.org/10.1182/blood. V118.21.1955.1955

23. Gluckman E, Cappelli B, Bernaudin F et al (2017) Sickle cell disease: an international survey of results of HLA-identical sibling hematopoietic stem cell transplantation. Blood 129:1548-1556. https:// doi.org/10.1182/blood-2016-10-745711

24. Goldbaum MH (1978) Retinal depression sign indicating a small retinal infarct. Am J Ophthalmol 86:45-55. https://doi.org/10.1016/00029394(78)90013-2

25. Goldberg MF (1971) Classification and pathogenesis of proliferative sickle retinopathy. Am J Ophthalmol 71:649-665.https://doi.org/10.1016/ 0002-9394(71)90429-6

26. Goldberg MF (1976) Retinal vaso-occlusion in sickling hemoglobinopathies. Birth Defects Orig Artic Ser 12:475-515

27. Goldberg MF (1978) The diagnosis and treatment of sickled erythrocytes in human hyphemas. Trans Am Ophthalmol Soc 76:481-501

28. Gragert L, Eapen M, Williams E et al (2014) HLA match likelihoods for hematopoietic stem-cell grafts in the U.S. registry. $\mathrm{N}$ Engl J Med 371:339-348. https://doi.org/10.1056/ NEJMsa1311707

29. Gubler M, Levy M, Broyer M et al (1981) Alport's syndrome. Am J Med 70:493-505. https://doi.org/ 10.1016/0002-9343(81)90571-4
30. Han IC, Tadarati M, Pacheco KD, Scott AW (2017) Evaluation of macular vascular abnormalities identified by optical coherence tomography angiography in sickle cell disease. Am JOphthalmol 177:90-99. https://doi.org/10.1016/j.ajo.2017.02. 007

31. Han IC, Tadarati M, Scott AW (2015) Macular vascular abnormalities identified by optical coherence tomographic angiography in patients with sickle cell disease. JAMA Ophthalmol 133:1337. https:// doi.org/10.1001/jamaophthalmol.2015.2824

32. Hayreh SS, Podhajsky PA, Zimmerman MB (2009) Branch retinal artery occlusion. Ophthalmology 116:1188-1194.e4. https://doi.org/10.1016/j. ophtha.2009.01.015

33. Hoang QV, Chau FY, Shahidi M, Lim JI (2011) Central macular splaying and outer retinal thinning in asymptomatic sickle cell patients by spectraldomain optical coherence tomography. Am J Ophthalmol 151:990-994.e1. https://doi.org/10. 1016/j.ajo.2010.12.010

34. Hussnain SA, Coady PA, Stoessel KM (2017) Paracentral acute middle maculopathy: precurso to macular thinning in sickle cell retinopathy. BMJ Case Rep. https://doi.org/10.1136/bcr-2016216124

35. Ikawa Y, Miccio A, Magrin E et al (2019) Gene therapy of hemoglobinopathies: progress and future challenges. Hum Mol Genet 28:R24-R30. https://doi.org/10.1093/hmg/ddz172

36. Inamoto Y, Petriček I, Burns L et al (2019) NonGVHD ocular complications after hematopoietic cell transplantation: expert review from the late effects and quality of life working committee of the CIBMTR and transplant complications working party of the EBMT. Bone Marrow Transplant 54:648-661. https://doi.org/10.1038/s41409018-0339-6

37. Kohne E, Kleihauer E (2010) Hemoglobinopathies: a longitudinal study over four decades. Dtsch Arztebl Int 107:65-71. https://doi.org/10.3238/ arztebl.2010.0065

38. Kunz JB, Lobitz S, Grosse R et al (2020) Sickle cell disease in Germany: results from a national registry. Pediatr Blood Cancer 67:e28130. https:// doi.org/10.1002/pbc.28130

39. Lana-Peixoto MA, Barbosa A (1998) Anterior ischaemic optic neuropathy in a child with AS haemoglobinopathy and migraine. $\mathrm{Br} J$ Ophthalmol 82:196d. https://doi.org/10.1136/bjo. 82.2.196d

40. Leffler CT, Bharucha G, Donaldson J (2016) Management of hyphema in patients with sickle cell disease or trait. Surv Ophthalmol 61:689-690. https://doi.org/10.1016/j.survophthal.2016.04. 001

41. Leung CKS, Tham CCY, Mohammed S et al (2007) In vivo measurements of macular and nerve fibre layer thickness in retinal arterial occlusion. Eye (Lond) 21:1464-1468. https://doi.org/10.1038/sj. eye. 6702457

42. Leveziel N, Bastuji-Garin S, Lalloum $F$ et al (2011) Clinical and laboratory factors associated with the severity of proliferative sickle cell retinopathy in patients with sickle cell hemoglobin C (SC) and homozygous sickle cell (SS) disease. Medicine 90:372-378. https://doi.org/10.1097/ MD.0b013e3182364cba

43. Magrath GN, Pulido JS, Montero J et al (2010) Cystoid macular edema secondary to fluconazole toxicity. Ocul Immunol Inflamm 18:472-474. https://doi.org/10.3109/09273948.2010.507320

44. Martin GC, Dénier C, Zambrowski O et al (2017) Visual function in asymptomatic patients with homozygous sickle cell disease and temporal macular atrophy. JAMA Ophthalmol 135:1100. https://doi.org/10.1001/jamaophthalmol.2017. 3008

45. Martin GC, Albuisson E, Brousse V et al (2019) Paramacular temporal atrophy in sickle cell disease occurs early in childhood. Br J Ophthalmol 103:906-910. https://doi.org/10.1136/ bjophthalmol-2018-312305

46. Mathew R, Bafiq R, Ramu J et al (2015) Spectral domain optical coherence tomography in patients with sickle cell disease. Br J Ophthalmol 99:967-972. https://doi.org/10.1136/ bjophthalmol-2014-305532

47. Mitropoulos PG, Chatziralli IP, Parikakis EA et al (2014) Intravitreal ranibizumab for stage IV proliferative sickle cell retinopathy: a first case report. Case Rep Ophthalmol Med 2014:682583. https://doi.org/10.1155/2014/682583

48. Modell B (2008) Global epidemiology of haemoglobin disorders and derived service indicators. Bull World Health Organ 2008:480-487. https:// doi.org/10.2471/BLT.06.036673

49. Moshiri A, Ha NK, Ko FS, Scott AW (2013) Bevacizumab presurgical treatment for proliferative sickle-cell retinopathy-related retinal detachment. Retin Cases Brief Rep 7:204-205. https://doi.org/ 10.1097/ICB.0b013e3182845d31

50. Murthy RK, Grover S, Chalam KV (2011) Temporal macular thinning on spectral-domain optical coherence tomography in proliferative sickle cell retinopathy. Arch Ophthalmol 129:247-249. https://doi.org/10.1001/archophthalmol.2010. 357

51. Nagpal KC, Asdourian G, Goldbaum M et al (1976) Angioid streaks and sickle haemoglobinopathies. Br J Ophthalmol 60:31-34. https://doi.org/10. 1136/bjo.60.1.31

52. Nakamura M, Katagiri S, Hayashi T et al (2019) Longitudinal follow-up of two patients with isolated paracentral acute middle maculopathy. Int Med Case Rep J 12:143-149. https://doi.org/10 2147/IMCRJ.S196047

53. Niihara Y, Miller ST, Kanter J et al (2018) A phase 3 trial of L-glutamine in sickle cell disease. $\mathrm{N}$ Engl J Med 379:226-235. https://doi.org/10.1056/ NEJMoa1715971

54. Pan Y, Keane PA, Sadun AA, Fawzi AA (2010) Optical coherence tomography findings in deferasirox-related maculopathy. Retin Cases Brief Rep 4:229-232. https://doi.org/10.1097/ICB. 0b013e3181af7b44

55. Panepinto JA, Walters MC, Carreras J et al (2007) Matched-related donor transplantation for sickle cell disease: report from the center for international blood and transplant research. $\mathrm{Br}$ Haematol 137:479-485. https://doi.org/10.1111/j. 1365-2141.2007.06592.x

56. Park HY-L, Kim IT, Park CK (2011) Early diabetic changes in the nerve fibre layer at the macula detected by spectral domain optical coherence tomography. Br J Ophthalmol 95:1223-1228. https://doi.org/10.1136/bjo.2010.191841

57. Paton D (1962) The conjunctival sign of sickle-cell disease: further observations. Arch Ophthalmol 68:627. https://doi.org/10.1001/archopht.1962. 00960030631010

58. Perlman JI, Forman S, Gonzalez ER (1994) Retrobulbar ischemic optic neuropathy associated with sickle cell disease. J Neuroophthalmo 14:45-48

59. Pinto VM, Balocco M, Quintino S, Forni GL (2019) Sickle cell disease: a review for the internist. Intern 
Emerg Med 14:1051-1064. https://doi.org/10. 1007/s11739-019-02160-x

60. Platt OS, Orkin SH, Dover Getal (1984) Hydroxyurea enhances fetal hemoglobin production in sickle cell anemia. J Clin Invest 74:652-656. https://doi. org/10.1172/JCl111464

61. Rahimy E, Kuehlewein L, Sadda SR, Sarraf D (2015) Paracentral acute middle maculopathy: what we knew then and what we know now. Retina 35:1921-1930. https://doi.org/10.1097/ IAE. 0000000000000785

62. Rodrigues M, Kashiwabuchi F, Deshpande M et al (2016) Expression pattern of HIF-1 $a$ and VEGF supports circumferential application of scatter laser for proliferative sickle retinopathy. Invest Ophthalmol Vis Sci 57:6739-6746. https://doi.org/ 10.1167/iovs.16-19513

63. Romayanada N, Goldberg MF, Green WR (1973) Histopathology of sickle cell retinopathy. Trans Am Acad Ophthalmol Otolaryngol 77:OP652-676

64. Rupp-Montpetit K, Moody ML (2005) Visual loss as a complication of non-ophthalmic surgery: a review of the literature. Insight 30:10-17

65. Sambhav K, Grover S, Chalam KV (2017) Temporal thinning in sickle cell retinopathy is associated with diminished perfusion on octa and dense scotoma on microperimetery. Retin Cases Brief Rep. https:// doi.org/10.1097/ICB.0000000000000610

66. Savige J, Liu J, DeBuc DC et al (2010) Retinal basement membrane abnormalities and the retinopathy of Alport syndrome. Invest Ophthalmol Vis Sci 51:1621. https://doi.org/10.1167/iovs.083323

67. Savige J, Sheth S, Leys A et al (2015) Ocular features in Alport syndrome: pathogenesis and clinical significance. Clin J Am Soc Nephrol 10:703-709. https://doi.org/10.2215/CJN.10581014

68. Slagle WS, Musick AN, Eckermann DR (2009) Diabetic papillopathy and its relation to optic nerve Ischemia. Optom Vis Sci 86:e395-e403. https://doi.org/10.1097/OPX.0b013e318198927c

69. Slavin ML, Barondes MJ (1988) Ischemic optic neuropathy in sickle cell disease. Am J Ophthalmol 105:212-213. https://doi.org/10.1016/00029394(88)90191-2

70. Stevens TS, Busse B, Lee CB et al (1974) Sickling hemoglobinopathies; macular and perimacular vascular abnormalities. Arch Ophthalmol 92:455-463. https://doi.org/10.1001/archopht. 1974.01010010469002

71. Switzer GE, Bruce JG, Myaskovsky L et al (2013) Race and ethnicity in decisions about unrelated hematopoietic stem cell donation. Blood 121:1469-1476. https://doi.org/10.1182/ blood-2012-06-437343

72. Thavikulwat AT, Cao D, Vajaranant TS, Lim JI (2019) Longitudinal study of peripapillary thinning in sickle cell hemoglobinopathies. Am J Ophthalmol 202:30-36. https://doi.org/10.1016/j.ajo.2019.02. 006

73. Torres L, Conran N (2019) Emerging pharmacotherapeutic approaches for the management of sickle cell disease. Expert Opin Pharmacother 20:173-186. https://doi.org/10.1080/14656566. 2018.1548610

74. Usui T, Ichibe M, Hasegawa $S$ et al (2004) Symmetrical reduced retinal thickness in a patient with Alport syndrome. Retina 24:977-979. https:// doi.org/10.1097/00006982-200412000-00026

75. Walia HS, Yan J (2013) Reversible retinopathy associated with oral deferasirox therapy. BMJ Case Rep 2013:bcr2013009205. https://doi.org/ 10.1136/bcr-2013-009205
76. Ware RE, de Montalembert $M$, Tshilolo $L, A b$ boud MR (2017) Sickle cell disease. Lancet 390:311-323. https://doi.org/10.1016/S0140 6736(17)30193-9

77. Welch RB, Goldberg MF (1966) Sickle-cell hemoglobin and its relation to fundus abnormality. Arch Ophthalmol 75:353-362. https://doi.org/10.1001/ archopht.1966.00970050355008

78. Williamson TH, Rajput R, Laidlaw DAH, Mokete B (2009) Vitreoretinal management of the complications of sickle cell retinopathy by observation or pars plana vitrectomy. Eye (Lond) 23:1314-1320. https://doi.org/10.1038/eye.2008.296

\section{Terminankündigung}

\section{2}

AAD 2022 hybrid

Düsseldorf, 16.03. - 19.03.2022

https://aad-kongress.de/

Jahrestagung der BielschowskyGesellschaft 2022

Freiburg i.B., 13.05. - 14.05.2022

http://www.bg2022.de/

DOG 2022

Berlin, 29.09. - 02.10.2022

https://dog-kongress.de/

2023

AAD 2023

Düsseldorf, 21.03. - 25.03.2023

https://aad-kongress.de/

DOG 2023

Berlin, 28.09. - 01.10.2023

https://dog-kongress.de/

2024

AAD 2024

Düsseldorf, 12.03. - 16.03.2024

https://aad-kongress.de/

DOG 2024

Berlin, 10.10. - 13.10.2024

https://dog-kongress.de/ 